\title{
Roi sorcier, mère sorcière. Parenté, politique et sorcellerie en Afrique noire
}

Paris, Éditions du Félin, 2006, 248 p.Julien Bonhomme

URL : http://journals.openedition.org/assr/3833

DOI : $10.4000 /$ assr.3833

ISSN : $1777-5825$

Éditeur

Éditions de l'EHESS

Édition imprimée

Date de publication : 1 décembre 2006

ISBN : 2-7132-2124-2

ISSN : 0335-5985

Référence électronique

"Roi sorcier, mère sorcière. Parenté, politique et sorcellerie en Afrique noire », Archives de sciences sociales des religions [En ligne], 136 | octobre - décembre 2006, document 136-2, mis en ligne le 03 décembre 2013, consulté le 21 septembre 2020. URL : http://journals.openedition.org/assr/3833 ; DOI : https://doi.org/10.4000/assr.3833

Ce document a été généré automatiquement le 21 septembre 2020.

(C) Archives de sciences sociales des religions 


\section{Roi sorcier, mère sorcière. Parenté, politique et sorcellerie en Afrique noire}

Paris, Éditions du Félin, 2006, 248 p.Julien Bonhomme

1 Cet ouvrage d'anthropologie s'intéresse à l'articulation entre sorcellerie et rapports de pouvoir en Afrique noire. Comme le note en effet l'auteur, "partout où elle s'exerce, c'est sur le terrain des rapports de force que la sorcellerie donne la mesure de sa réalité » (p. 9). La notion de «force » constitue ainsi le pivot de l'analyse, dans la mesure où elle permet l'articulation entre le discours indigène (évoquant les «forces occultes " manipulées par le sorcier pour nuire à autrui) et l'explication savante (en termes de rapports de force et de pouvoir).

2 Afin de mettre au jour ces liens entre sorcellerie et pouvoir, l'ouvrage s'intéresse moins aux représentations symboliques qu'aux protagonistes des affaires de sorcellerie (victimes et accusés notamment) et aux positions respectives qu'ils occupent dans l'organisation sociale. L'approche adoptée rejoint en cela la démarche fonctionnaliste des nombreux travaux de l'anthropologie sociale britannique consacrés à la sorcellerie africaine dans les années 1950 et 1960. En revanche, l'auteur se démarque des versions les plus radicales de l'explication fonctionnaliste et refuse de réduire la sorcellerie à n'être qu'un instrument au service de l'ordre social - reprenant ainsi à son compte les critiques adressées par Mary Douglas (M. D. éd., Witchcraft Confessions and Accusations, London, Tavistock Publications, 1970) à l'égard du finalisme sociologique des travaux sur la sorcellerie. Si la sorcellerie n'a pas de finalité sociale univoque, des rapports d'expression articulent néanmoins le système des accusations sorcellaires et le système sociopolitique. Ce sont ces rapports d'expression et leur variabilité qui sont l'objet central de ce travail.

3 L'organisation de l'ouvrage est fondée sur la typologie classique des systèmes sociopolitiques africains, typologie qui distingue les sociétés segmentaires (à pouvoir lignager) des chefferies et royautés (à pouvoir politique autonome). La première partie, «Sorcellerie et ordre social», est ainsi consacrée aux rapports entre sorcellerie et 
parenté dans les sociétés lignagères ; la seconde partie, « Pouvoir royal et sorcellerie ", aux rapports entre sorcellerie et politique dans les royautés et chefferies.

Il est notable que ces questions ont été déjà très largement travaillées en anthropologie. L'ouvrage renouvelle néanmoins ces travaux classiques, notamment grâce à un usage méthodique du comparatisme ethnologique. Alors que nombre d'auteurs ont voulu construire la figure exemplaire du parent-sorcier ou du roi-sorcier à partir d'un cas ethnographique particulier, Alfred Adler s'attache au contraire à faire ressortir la grande diversité des articulations entre sorcellerie, parenté et politique en Afrique noire. Modèle d'ethnologie comparative, son livre aurait ainsi tout aussi bien pu s'intituler "Variétés de la sorcellerie en Afrique noire ». On peut néanmoins regretter que les exemples relèvent majoritairement de l'Afrique de l'Ouest et de l'Afrique centrale, au détriment de l'Afrique de l'Est et de l'Afrique australe.

5 Par contraste avec la Mélanésie, l'Amazonie ou l'Europe, en Afrique noire le sorcier présumé est souvent un parent. L'ouvrage montre cependant la diversité des rapports entre sorcellerie et parenté (chap.2). Ce chapitre témoigne d'un recours très systématique et maîtrisé au comparatisme, puisqu'il examine successivement le lien de la sorcellerie à la filiation (Bakongo du Congo, Mesakin du Soudan) puis à l'alliance (Baluba de République démocratique du Congo, Thonga du Mozambique) avant d'envisager un exemple ethnographique articulant les deux registres au niveau de l'atome de parenté (Kuranko de Sierra Leone). Ce dernier cas évoque ainsi la figure d'une sœur accusée de sorcellerie, en quelque sorte sacrifiée parce qu'elle incarne la tension structurelle entre filiation et alliance. L'examen des rapports entre sorcellerie et filiation illustre bien le raffinement comparatiste : il s'agit toujours de procéder par variations systématiques en soulignant les différences structurelles. Si dans les sociétés matrilinéaires, le sorcier est souvent l'oncle utérin, les Bakongo jugent légitime que l'oncle «mange » son neveu (paradoxe d'une sorcellerie légitime car liée à l'autorité lignagère) alors que les Mesakin jugent au contraire légitime que le neveu accuse son oncle de sorcellerie.

6 Les chapitres 3 et 4 abordent l'épineuse question des raisons de la présence ou de l'absence de la sorcellerie dans des sociétés voisines. Reprenant des analyses de Meyer Fortes, l'auteur s'intéresse aux Tallensi du Ghana parmi lesquels la sorcellerie n'occupe qu'une faible place alors qu'elle est bien présente chez leurs voisins Ashanti (sous la figure d'une vieille femme envieuse dévorant son propre matrilignage). Chez les Tallensi, cette absence de la sorcellerie pourrait être due à l'omniprésence (protectrice mais parfois aussi punitive) des ancêtres. La présence envahissante des ancêtres ne laisserait donc guère de place aux sorciers dans l'explication du malheur (sanction légitime des ancêtres versus persécution injuste des sorciers).

7 Une autre piste d'explication amène l'auteur à examiner les conceptions du destin personnel dans plusieurs sociétés ouest-africaines. Si la prégnance de sorcellerie dépend de la place relative des ancêtres et des sorciers, elle dépend également des conceptions de la personne. Chez les Tallensi, la destinée individuelle (le yin) d'une personne offre en effet une explication possible au malheur qui esquive la sorcellerie et, par là, la question de la faute et de la culpabilité. Le malheur est provoqué par un «mauvais yin» dont personne n'est responsable mais qu'un rituel de réparation permet de rectifier. De même chez les Samo du Burkina Faso, le destin individuel (lepere) permet d'absoudre le sorcier, qui est considéré comme le simple instrument du lepere de sa victime. 
Quitter les sociétés lignagères pour les chefferies et royaumes africains permet de passer de la figure du parent-sorcier à celle du roi-sorcier. Ce personnage paradoxal fournit à l'auteur l'occasion d'une réflexion sur l'ambivalence du pouvoir et de la sorcellerie en Afrique noire. Si la sorcellerie est fondamentalement liée à la question du mal, ce lien n'est pas dénué d'ambiguïté : « on peut affirmer, d'une manière générale, que les idéologies relevant d'une pensée manichéenne (au sens vulgaire du terme) ne se rencontrent guère en Afrique noire » (p. 27). En effet, le sorcier est un être méchant, mais il est aussi un homme fort. Tout homme de pouvoir est d'ailleurs virtuellement lui aussi un sorcier. Et le contre-sorcier lui-même est souvent perçu comme un sorcier (comme l'affirme sans ambages un proverbe des Bapunu du Gabon, « le guérisseur et le sorcier sont des amis »). Ces représentations semblent bien témoigner d'une certaine méfiance et d'un certain pessimisme à l'égard du pouvoir. Ce " paradoxe du pouvoir » a déjà été souligné par nombre d'auteurs (par exemple, Philippe Laburthe-Tolra, Initiations et sociétés secrètes au Cameroun, Paris, Karthala, 1985 ; Peter Geschiere, Sorcellerie et politique en Afrique, Paris, Karthala, 1995). Mais Alfred Adler montre comment cette ambivalence du pouvoir et de la sorcellerie se condense sous une forme paradoxale dans la figure énigmatique du roi-sorcier.

En effet, le roi-sorcier concentre dans sa personne la figure du sorcier criminel et celle du souverain légitime justement censé être un rempart protecteur contre la sorcellerie. Partant des analyses de Luc de Heusch sur le roi kongo (qui part lui-même des analyses de James Frazer sur le roi-magicien), Alfred Adler réexamine ce paradoxe du roi-sorcier à partir d'une large matrice comparatiste qui couvre l'Afrique centrale (chap. 5) mais aussi l'Afrique de l'Ouest (chap.6) (cf. James Frazer, Le Rameau d'or, Paris, Robert Laffont, 1981-1983 ; Luc de Heusch, Le Roi de Kongo et les monstres sacrés, Paris, Gallimard, 2000). Ce réexamen offre en outre l'occasion d'une critique des distinctions conceptuelles - déjà fort anciennes - entre religion, magie et sorcellerie. Comme le souligne l'auteur, la dichotomie entre magie et religion est plus complexe que l'opposition entre l'individuel et le collectif, de même que la dichotomie entre magie et sorcellerie est plus complexe que l'opposition entre le social et l'antisocial.

L'auteur commence par passer en revue un certain nombre de figures royales de l'aire bantu d'Afrique centrale (Tio du Congo, Kuba de République démocratique du Congo, Nyakusa du Malawi). Il relève ainsi que le roi tio associé au lion, le roi kuba associé au léopard et le roi nyakusa associé au python incarnent tous trois la figure du roi-sorcier bien que sur des modes différents. Ces royautés d'Afrique centrale illustrent ainsi de façon exemplaire que la contre-sorcellerie est encore une forme de sorcellerie. L'examen de rois-sorciers d'Afrique occidentale permet de compliquer ce modèle en introduisant de nouvelles variations. Chez les Songhay du Niger, le contre-sorcier se rapproche du sorcier en raison de l'affirmation de sa filiation mythique avec le souverain magicien Sonni Ali. Chez les Yoruba du Nigeria, le souverain a besoin de l'appui de la "mère des sorcières », grande prêtresse de l'orisa aquatique Yemoja, afin de revitaliser périodiquement son pouvoir. Par contraste avec l'aire bantu, la sorcellerie est dans ce cas très liée au pouvoir des femmes commerçantes. Les Gonja du Ghana opposent une sorcellerie féminine illégitime (dans la sphère domestique) et une sorcellerie masculine légitime dans la sphère politique de luttes de pouvoir au sein d'une fédération de chefferies. Enfin, les Tiv du Nigeria offrent l'exemple d'une société "furieusement égalitaire » qui se constitue pourtant en royauté à la fin du XIX siècle sous la pression expansionniste des populations voisines. C'est alors l'organe de 
sorcellerie tsav qui sert de fondement à cette nouvelle royauté reposant sur une idéologie sacrificielle et cannibale.

11 À travers une série systématique de variations qui n'est pas sans évoquer un modèle musical, l'ouvrage passe ainsi en revue l'étonnante diversité des rapports entre sorcellerie, parenté et politique dans les sociétés d'Afrique noire. Á l'issue d'une telle lecture, on est convaincu que l'anthropologie sociale britannique avait raison de placer l'étude de la sorcellerie sous l'angle de ses rapports avec l'organisation sociale, mais également qu'elle avait tort de penser ces rapports de manière trop rigidement déterministes. La sorcellerie constitue en définitive un registre de choix pour décrypter «les relations réelles de pouvoir qui se cachent, plus ou moins et parfois presque pas du tout, sous le manteau des institutions politiques officielles » (p. 227).

On peut néanmoins regretter que l'ouvrage s'appuie sur des matériaux ethnographiques souvent déjà anciens et sur une littérature parfois un peu datée. Rien n'est dit des transformations contemporaines de la sorcellerie. Les liens avec la parenté et la politique sont-ils maintenus, rompus, déplacés? Seule la toute dernière page de l'ouvrage annonce rapidement qu'« avec l'État moderne, pas plus qu'avec la chefferie ou la royauté, on ne sort du cercle enchanté de la sorcellerie» (p. 235). Il existe pourtant une littérature aujourd'hui très fournie sur la "modernité sorcellaire » en Afrique (Par exemple, Jean \& John L. Comaroff, eds., Modernity and its Malcontents. Ritual and power in postcolonial Africa, 1993; Peter Geschiere, Sorcellerie et politique en Afrique, 1995; Henrietta Moore \& Todd Sanders, eds., Magical Interpretations, Material Realities. Modernity, witchcraft and the occult in postcolonial Africa, 2001 ; Birgit Meyer \& Peter Pels, eds., Magic and modernity. Interfaces of revelation and concealment, 2003; Adam Ashforth, Witchcraft, Violence, and Democracy in South Africa, 2005; Harry West, Kupilikula. Governance and the invisible realm in Mozambique, 2005 ; Joseph Tonda, Le souverain moderne. Le corps du pouvoir en Afrique centrale (Congo, Gabon), 2005). L'ouvrage passe ainsi sous silence les articulations complexes entre sorcellerie et pouvoir politique dans le cadre de l'État colonial puis postcolonial. Rien n'est dit non plus des innombrables prophétismes et mouvements anti-sorcellerie, et de la façon dont ils ont tenté - certes souvent en vain - de modifier les liens entre sorcellerie, parenté et politique (Voir JeanPierre Dozon, La cause des prophètes. Politique et religion en Afrique contemporaine, Paris, Seuil, 1995 ; André Mary, Le défi du syncrétisme, Le travail symbolique de la religion d'eboga (Gabon), Paris, EHESS, 1999 ; John Cinnamon, « Ambivalent Power. Anti-Sorcery and Occult Subjugation in Late Colonial Gabon », Journal of Colonialism and Colonial History, 3-3, 2002 revue en ligne). De même, les transformations de la question du mal sous l'influence du christianisme missionnaire (ou de l'islam) ne sont pas évoquées : on imagine pourtant bien que ces phénomènes affectent la sorcellerie et sont affectés en retour par elle (Birgit Meyer, Translating the devil. Religion and modernity among the Ewe in Ghana, Trenton, Africa World Press, 1999 ; André Mary, «La diabolisation du sorcier et le réveil de Satan ", Religiologiques, 1998, $\mathrm{n}^{\circ}$ 18, p. 53-77 ; Joseph Tonda, La guérison divine en Afrique centrale (Congo, Gabon), Paris, Karthala, 2002). Il est dommage de laisser tous ces faits sociaux hors champ dans la mesure où la démarche adoptée est comparatiste et opère par variations systématiques. La prise en compte des dynamiques contemporaines aurait ainsi pu permettre d'évoquer la sortie de la sorcellerie hors de la sphère de la parenté (par exemple, la focalisation des accusations sorcellaires sur des communautés allochtones) ou encore de mettre au jour des évolutions d'ensemble qui dépassent bien souvent les particularismes ethniques et régionaux (par exemple, la focalisation des accusations sorcellaires sur les «nouveaux riches", les hommes 
politiques ou les fonctionnaires de l'État postcolonial). Cela aurait en définitive permis d'enrichir avec bonheur cette étude - certes déjà fort riche - de la sorcellerie africaine et de ses articulations sociopolitiques. 